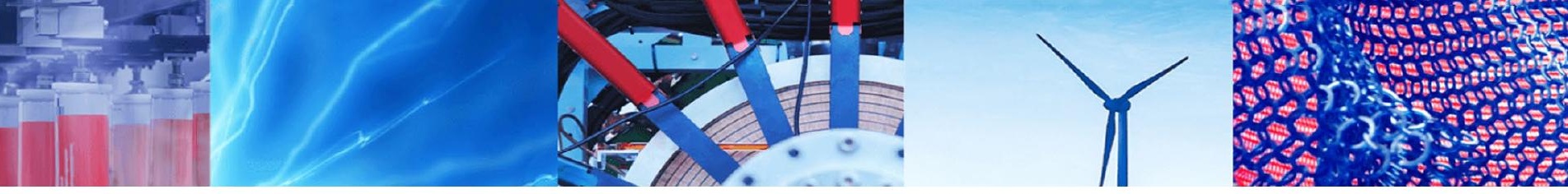

Research Article

\title{
Processing natural wood into bulk conducting materials
}

\author{
Jun-Ye Zhang ${ }^{1} \cdot$ Qing Shen ${ }^{1}$ (D) \\ Received: 6 June 2019 / Accepted: 24 October 2019 / Published online: 9 November 2019 \\ (c) Springer Nature Switzerland AG 2019
}

\begin{abstract}
By taking the natural bulk wood segments to immerse in the aniline, ANI, solution to allow the wood pores swelling and ANI molecules penetration or diffusion, initially, and then to in situ synthesize the polyaniline, PANI, within wood, we produced the bulk conducting wood. Results showed that this bulk conducting wood has conductivity at about $0.13 \mathrm{~S} /$ $\mathrm{cm}$ and an enhanced thermal stability as comparing to the referenced pure wood. The mechanism on formation of the bulk conducting wood has been found due to the ANI molecules initially pre-penetrated or diffused into the wood pore structure to fill and coat the pores to lead the subsequent chemical interactions occurred between the NHs groups of ANI and the $\mathrm{CH}_{2}-\mathrm{OH}$ groups of wood through the glucose units of cellulose.
\end{abstract}

Keywords Bulk conducting wood $\cdot$ Polyaniline $\cdot$ Structure $\cdot$ Properties

\section{Introduction}

Wood is a traditional biomaterial with cellular structure [1] and has been long time widely studied and used for building and furniture construction. Very recently, some reports showed that the common wood can be treated as a high-strength material [2] or developed as wood-based functional materials [3-7].

With recent great growth in the application of electronic equipment, some unwanted and unexpected problems are revealed and even harmful electromagnetic interference. In some places, especially the cinema, concert, and operation room of hospital, the indoor decoration materials are expected to apply the electric signal shielded non-metal materials. This thus leads to some new developments in production of conductive composite materials for applying to preventing or reducing those mentioned problems. Several researchers have made attempts to produce the conducting wood [8-20]. Observed that all these reported cases only formed wood veneer or milled wood particles-based conducting materials without the fully bulk conducting wood. Notably, Kanwal et al. [18] have synthesized the typical conducting polymer, polyaniline, PANI, with milled wood to form the conducting wood board. Though this product can fit some applications, the bulk conducting wood is still required because wood is a heterogeneous bio-composite primarily composed of cellulose, lignin, hemicelluloses, and some ashes hierarchically organized within a cellular structure [21-23]. In fact, the bulk conducting wood has considerably advantages in keeping the cellular structure while in conductivity that the milled or veneer sample impossible.

Since yet without any report on bulk conducting wood, the aim of this work is proposed to take the bulk wood segments as samples to fabricate the bulk conducting wood. Experimentally, the wood segments were immersed in the ANI solution to allow the wood pores swelling to benefit the ANI molecules diffusion, then the in situ PANI synthesis was started [24-27] in the presence of bulk wood segments. The obtained bulk conducting wood was characterized by FESEM, FTIR, XRD, TG, conductivity, and water adsorption and compared with the referenced pure wood.

Qing Shen, sqing@dhu.edu.cn | ${ }^{1}$ State Key Laboratory for Modification of Chemical Fiber and Polymer Materials, Polymer Department of Donghua University, 2999 N. Renmin Rd., Songjiang, Shanghai 201620, People's Republic of China. 


\section{Materials and methods}

\subsection{Materials}

The birch wood segments with round rod were used by purchasing from a local market at Shanghai. These wood segments have an average diameter at about $5 \mathrm{~mm}$ and length at about $5 \mathrm{~cm}$ parallel to the fiber growth direction. The moisture of these wood segments was at about $3 \%$.

As previously described [24-27], the used aniline, ammonium peroxodisulfate, APS, and the solvents all in analytical grade reagents and obtained from the Sinopharm Chemical Reagent Co., Ltd., Shanghai, China and used without furthermore purification.

Lab made distilled water was always used through this work.

\subsection{Fabrication of bulk conducting wood}

The bulk conducting wood was fabricated using below steps. Initially, $0.05 \mathrm{~mol}$ ANI was added to $100 \mathrm{~mL} \mathrm{HCl}$ $(0.01 \mathrm{M})$ solution under magnetic stirring condition for 10 min to form an $\mathrm{ANI} / \mathrm{HCl}$ solution when one bulk wood segment was immersed for swelling about $4 \mathrm{~h}$ at $25^{\circ} \mathrm{C}$ to allow the ANI molecules easily penetration/diffusion into the wood capillary or other pores. Meanwhile, $11.41 \mathrm{~g}(0.05 \mathrm{~mol})$ APS was dissolved in $50 \mathrm{~mL}$ distilled water acting as the oxidizing and protonating agent to slowly add into the $\mathrm{ANI} / \mathrm{HCl}$ solution with bulk wood segment by keeping the time at about $30 \mathrm{~min}$ to avoid the temperature rapidly increase under a magnetic stirring condition until the color gradually changed from milk to green then in dark green (emeraldine) finally [24-27].

After polymerization processing to about $24 \mathrm{~h}$, the synthesis was stopped and the wood segment was washed by acetone and water alternatively and each in three times, respectively, then vacuum oven dried at $50^{\circ} \mathrm{C}$ for $24 \mathrm{~h}$.

During this work, three unique syntheses were parallel performed and each with only one wood sample.

\subsection{Characterization}

The field emission scanning electron microscopy (FESEM) images were prepared by drop casting an aqueous dispersion $(\sim 1 \mathrm{~g} / \mathrm{L})$ of the doped substituted sample onto a silicon wafer. A JEOL JSM-6700-F field emission SEM microscope was employed.

The Fourier transform infrared, FTIR, spectrum was recorded using the NEXUS 8700 (Nicolet, UK) in the range of $400-4000 \mathrm{~cm}^{-1}$ with the resolution of $4 \mathrm{~cm}^{-1}$. The $\mathrm{KBr}$ pellet technique was adopted to prepare all samples.

The X-ray diffraction, XRD, curves were recorded by the Rigaku D/Max-2550 PC instrument (Rigaku, USA) at $40 \mathrm{kV}, 30 \mathrm{~mA}$, by the Cu-Ka monochromatic radiation with a wavelength of $1.5406 \AA$, after scanning in the $2 \theta$ range of $3-60^{\circ}$ at intervals of 0.02 . For this measurement, the crystallinity values can be given by associated software.

The electrical conductivity was measured using SDY-4 Four-Point Probe Meter (Four Dimensions, Inc. USA) at $25^{\circ} \mathrm{C}$.

The thermal stability was characterized by the NETZSCH STA-409PC (NETZSCH, Germany) from 25 to $900^{\circ} \mathrm{C}$ with a heating rate of $10^{\circ} \mathrm{C} / \mathrm{min}$ under $\mathrm{N}_{2}$ atmosphere.

The water adsorption was performed at $25{ }^{\circ} \mathrm{C}$ by immersing the wood in water to measure its weight varied with time increase and meanwhile to estimate the porosity of wood samples on the basis of Eq. (1) $[28,29]$.

$P=\left[\left(m_{\text {wet }}-m_{\text {dry }}\right) /\left(m_{\text {air }}-m_{\text {dry }}\right)\right] \times 100 \%$,

where $P$ is the porosity (\%), $m$ is the weight of wood sample, and the subscripts represent the wood sample in dry, air, and wet, respectively.

During this measurement, the dried wood was weighed after oven drying about $24 \mathrm{~h}$ at $60^{\circ} \mathrm{C}$. The weight of wet saturated wood was measured after hot water $\left(100^{\circ} \mathrm{C}\right)$ immersion of $2 \mathrm{~h}$ then cooling to $25^{\circ} \mathrm{C}$. The weight of air saturated wood was measured by moving saturated water from wood using centrifuge.

\section{Results and discussions}

\subsection{Morphology of bulk conducting wood}

The FESEM images of the cross section of pure and bulk conducting wood were showed in Fig. 1, where the photographs of both the pure and conducting wood placed as inset, respectively. Comparing to the pure wood rod with natural color, Fig. 1 inset showed that the bulk conducting wood rod has fully dark color corresponding to the emeraldine state of PANI. This indicated that the PANI has been successfully in situ synthesized in wood pores and surface.

In terms of Fig. 1 left, the cross section of pure wood showed an enlarged image inset where a lot of open pores appeared corresponding to the capillary, while the bulk conducting wood (Fig. 1 right) showed that the most pores have been filled of PANI. This result is supported by literature because the wood has cellular structure that would be filled with in situ polymerized materials [30-33]. 


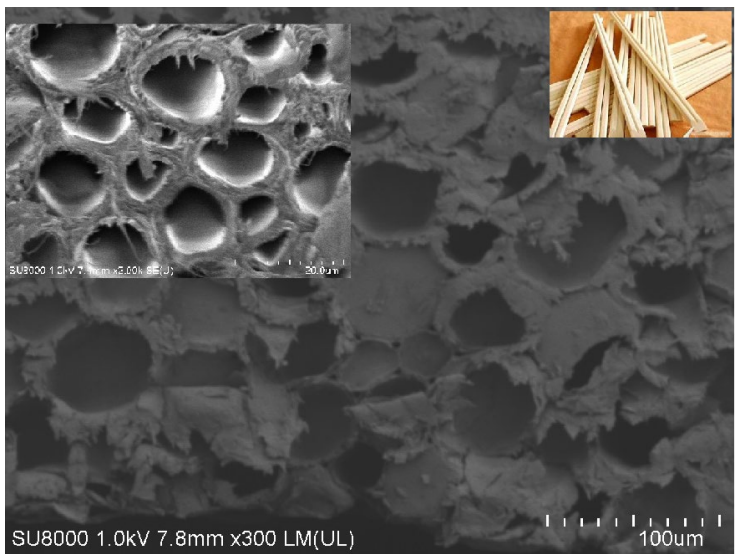

Pure wood

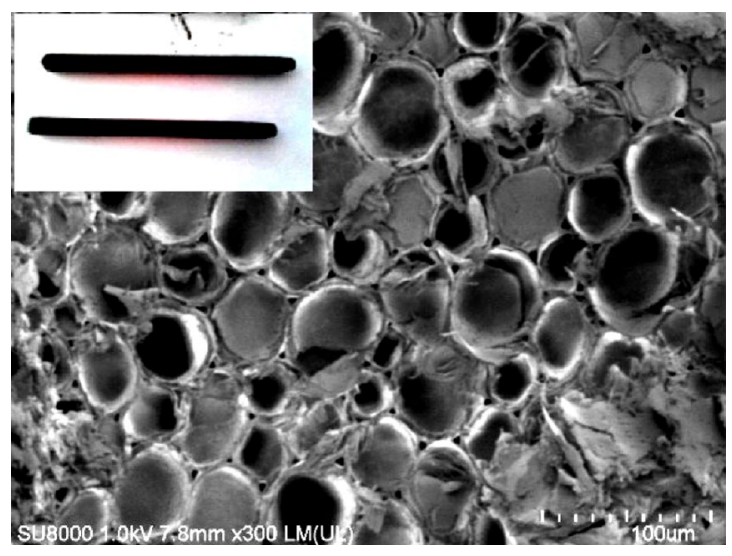

Bulk conducting wood

Fig. 1 FEEM images of the cross section of pure and bulk conducting wood, where the photographs of both pure and bulk conducting wood were placed as inset

Table 1 Basic parameters of pure and bulk conducting wood samples

\begin{tabular}{lllll}
\hline Samples & Conductivity $(\mathrm{S} / \mathrm{cm})$ & Crystallinity (\%) & Porosity (\%) & References \\
\hline Pure wood & 0 & 39.7 & 24.7 & This work \\
Pure PANI & 0.20 & 32.6 & - & This work \\
Bulk conducting wood & 0.13 & 45.8 & 15.0 & This work \\
Milled wood & 0.60 & - & - & {$[18]$} \\
Milled wood & 1.92 & - & - & {$[18]$} \\
Veneer & 0.0001 & - & - & {$[20]$} \\
Veneer & $0.04-0.40$ & - & - & {$[31]$} \\
\hline
\end{tabular}

According to the weight of wood sample measured before and after polymerization, the wood rod has increased the weight of $0.06 \pm 0.02 \mathrm{~g} / \mathrm{cm}^{3}$. Obviously, this implied the formation of wood/PANI composite. This was also proven by comparison of the porosity for both the pure and bulk conducting wood as presented in Table 1, because about $40 \%$ pores have been filled by PANI for this wood. This is importantly for producing conducting wood and reasonably supported by Trey et al. [20] and Hassel et al. [31] reported values because they have found about $10 \%$ PANI loaded in the wood veneers.

\subsection{Structure of bulk conducting wood}

The structure of bulk conducting wood was characterized by FTIR spectroscopy by taking the pure wood and PANI as references [24-27]. In the FTIR spectrum of conducting wood (Fig. 2), some intense bands located at 1736, 1622, 1040,895 , and $601 \mathrm{~cm}^{-1}$ are contributed by the lignin ring structure [34] suggesting the formed PANI within wood without changing the lignin-based wood structure.

Since the conducting wood showed a new band located at about $1260 \mathrm{~cm}^{-1}$ corresponding to the $\mathrm{C}=\mathrm{N}$ structure,

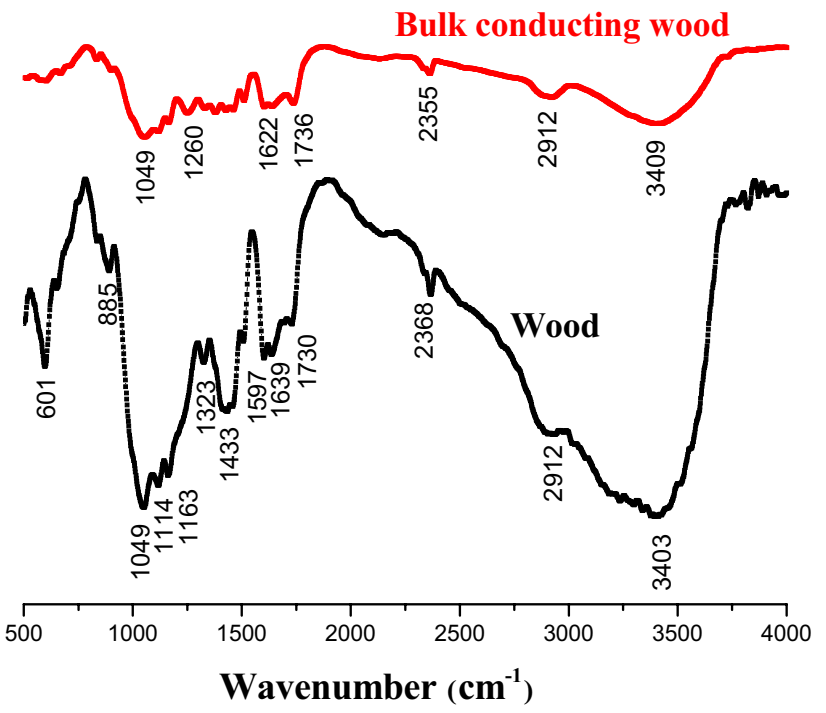

Fig. 2 FTIR spectra of the pure and bulk conducting wood

this importantly indicated that the pre-diffused $\mathrm{NH}_{2}$ groups of ANI within wood pores have interacted with the $\mathrm{CH}_{2}$ groups of wood by polymerization and formed 
the PANI/wood composite structure. This implied that the PANI molecules are not only physically filled the pores of wood but also chemically interacted with the functional groups of wood.

This is possible because Qaiser et al. [32] have found that the PANI molecules can be easily deposited on different places of structural materials, and this was furthermore proven by Trey et al. [20] and Hassel et al. [31], respectively.

The XRD patterns of pure and bulk conducting wood were compared by Fig. 3. A comparison found that the

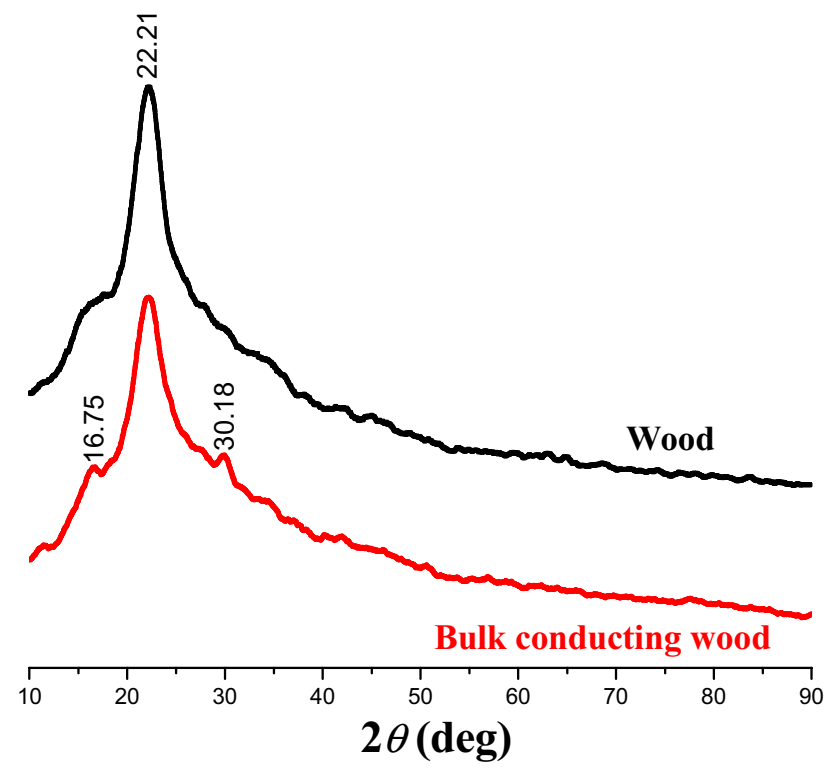

bulk conducting wood and pure wood both showed a typical peak at $2 \theta=22.21^{\circ}$ corresponding to the cellulose structure of (002), while the bulk conducting wood has also presented two new peaks located at $2 \theta=16.75^{\circ}$ and $30.18^{\circ}$ (Fig. 3) corresponding to the (101) and (040) of cellulose crystal structure [30,35]. This thus importantly indicated that the PANI molecules interacted with wood is mainly on the cellulose-based functional groups, e.g., from the $\beta(1 \rightarrow 4)$ linked $D$-glucose units [21-23].

Therefore, it is clearly understood that the synthesis of PANI within wood has caused the interactions between the $\mathrm{NH}_{2}$ groups of PANI and the $\mathrm{CH}_{2}-\mathrm{OH}$ groups in the glucose units of cellulose. A related reaction mechanism including the whole process was described in Fig. 4.

The bulk wood was initially swollen in ANI solution to allow the ANI molecules easily penetrated into the wood pores, then the polymerization was in situ started to induce the interactions occur between the $\mathrm{NH}_{2}$ groups of $\mathrm{ANI}$, and the $\mathrm{CH}_{2}-\mathrm{OH}$ groups of wood though the bridge of glucose units of cellulose to finally form the bulk conducting wood.

This was also proven by Table 1 presented crystallinity where the bulk conducting wood showed an enhanced value greater about $15 \%$ than that of the pure wood. This result is expected because this indicates that the electric charges can be easily moved to the side chains to enhance the conductivity of wood.

Fig. 3 XRD patterns of the pure and bulk conducting wood

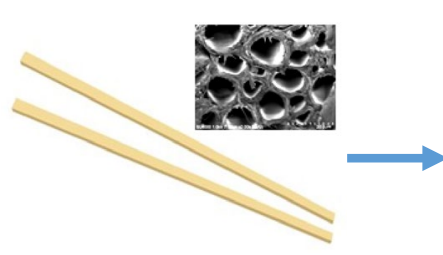

Original wood segments

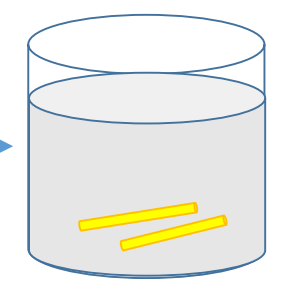

Wood swelling

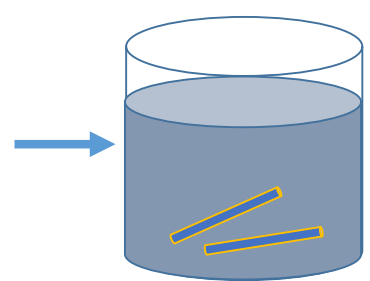

ANI molecules penetrated
APS adding

Solution polymerization

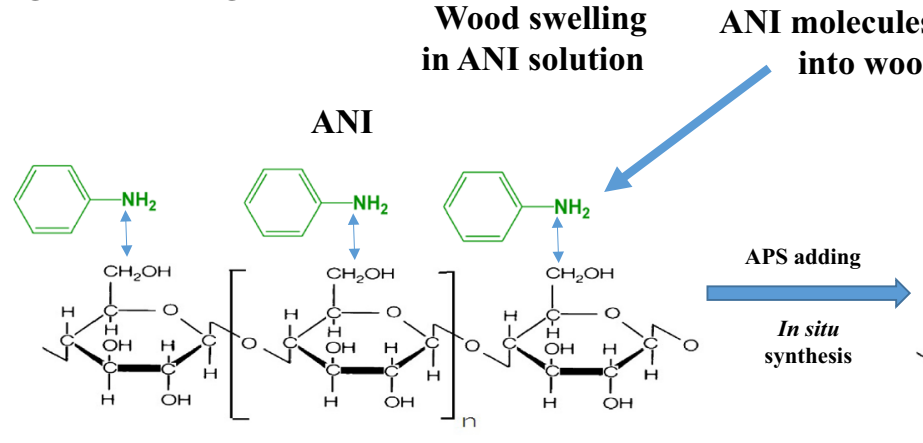

Cellulose structure of wood

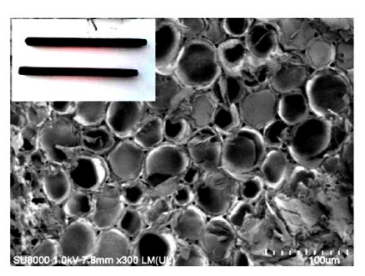

Conducting wood
PANI

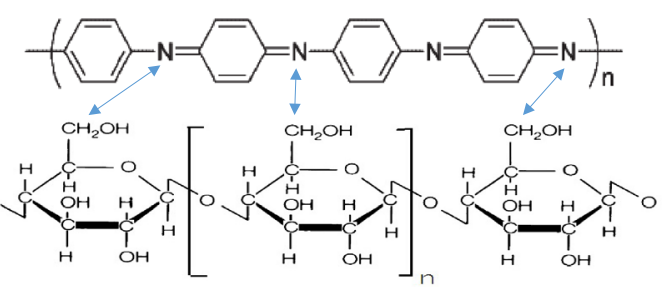

Cellulose structure of wood

Fig. 4 Scheme on processing the bulk conducting wood and related mechanism 


\subsection{Conductivity and other properties of bulk conducting wood}

Conductivity is an important parameter for electric materials [30]. For obtained bulk conducting wood, its conductivity was measured and compared with the parallel prepared pure wood and PANI as summarized in Table 1 where literature reported values on different conducting wood samples $[18,20,31]$ also appeared as references. Observe the conductivity of bulk conducting wood is visibly increased as compared with the referenced pure wood and supported by not only our prepared PANI samples as appeared in Table 1 but also previously reported values [24]. Since the conductivity of bulk conducting wood is smaller than that of the Kanwal et al. [18] reported values, we checked the synthesis temperature and found that Kanwal et al. [18] prepared the milled wood particlesbased wood board at 0 and $-5^{\circ} \mathrm{C}$ condition greatly lower than that of our employed $25^{\circ} \mathrm{C}$. As has been well known that the PANI synthesized at low temperature can increase the conductivity [18, 31, 34]. Additionally, the wood particles should be easily bonded with the ANI molecules to enhance the conductivity for wood particles-based board as compared with we prepared segment-based bulk conducting wood.

In fact, to see Trey et al. [20] and Hassel et al. [31] prepared conducting wood veneers is interested because the veneer sample has kept the some aspects of our bulk wood segment sample while these researchers reported conductivity on these wood veneers with a great varying range, e.g., within $0.04-0.40 \mathrm{~S} / \mathrm{cm}$. This thus implied that the PANI molecules bonded with the wood functional groups are heterogeneously for bulk wood and homogeneously for wood particles. From Table 1 presented conductivity value reported by Trey et al. [20] and Hassel et al. [31], the greater value is higher as compared with our bulk conducting wood even the referenced pure PANI sample. This is also reasonable because the thin veneer is somewhere easily diffused by ANI molecules to enhance the bonding between the ANI molecules with wood functional groups. Here, it should be addressed that the values reported by Trey et al. [20] and Hassel et al. [31] are impossible fully compared with our values (Table 1) because these researchers without presented parallel prepared pure PANI for comparison.

Figure 5 presents and compares the thermal stability of pure and conducting wood. It was found that the pure wood kept a good thermal stability when the temperature less about $300^{\circ} \mathrm{C}$ and the weight would be greatly reduced with the temperature increase over $300^{\circ} \mathrm{C}$. When the temperature continuously increase to about $600^{\circ} \mathrm{C}$, the pure wood again presented the good thermal stability by keeping its weight at about $30 \%$ corresponding to the carbon

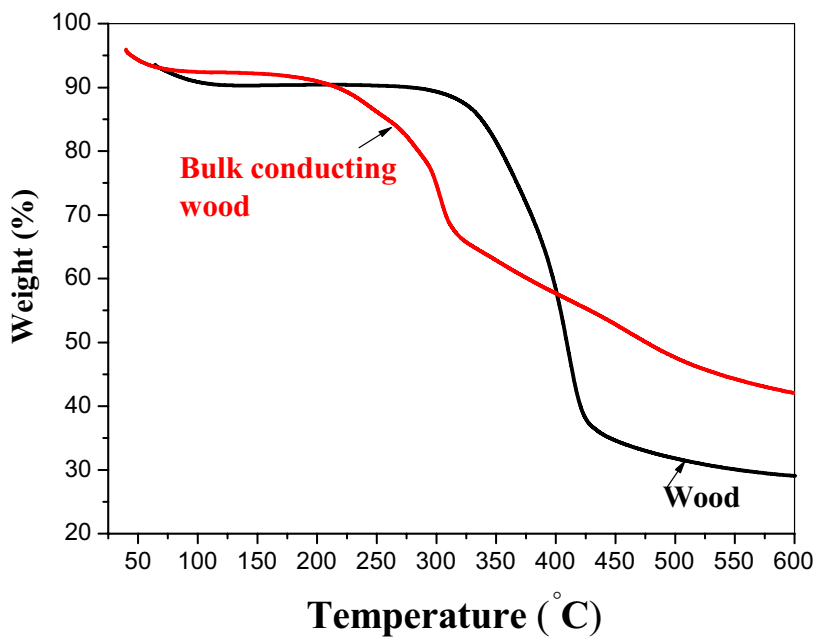

Fig. 5 TG curves of the pure and bulk conducting wood

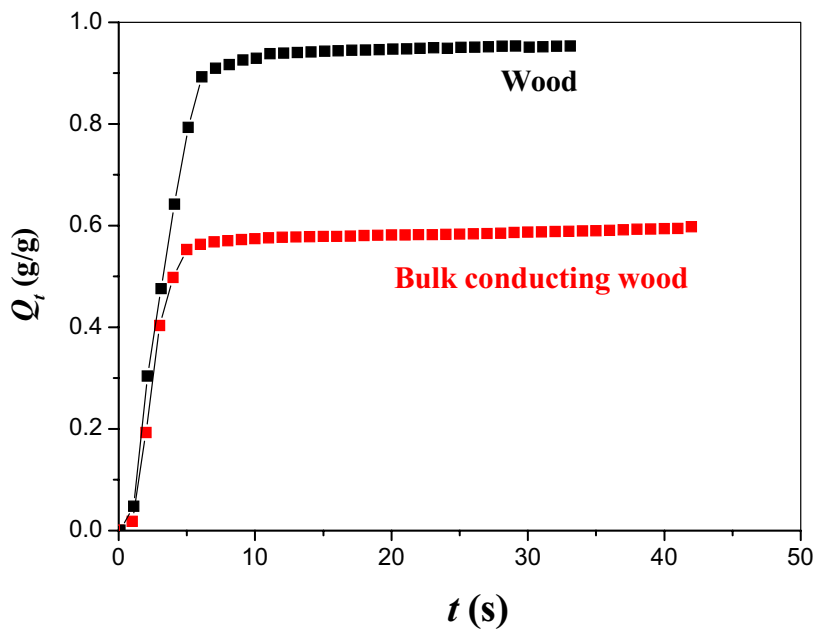

Fig. 6 Water adsorption behavior of the pure and bulk conducting wood

component-based structure. Comparing to the pure wood sample, the bulk conducting wood showed great weight reduction since the temperature up to about $200^{\circ} \mathrm{C}$, suggesting the thermal stability less than that of the pure wood in this temperature range. With the temperature furthermore increase up to over $300^{\circ} \mathrm{C}$, especially at about $400{ }^{\circ} \mathrm{C}$, the bulk conducting wood shown visible thermal stability enhancement than that of the pure wood because the bulk conducting wood kept the residue weight at about $41 \%$ that greater about $11 \%$ than the pure wood. This suggests that the bulk conducting wood is capable for applying as the indoor decoration materials.

The water adsorption kinetics was performed and compared for both the pure and bulk conducting wood as shown in Fig. 6. According to the adsorption, approaching 
to the equilibrium state resulted in adsorbed water amount, $Q_{e^{\prime}}$ at $0.58 \mathrm{~g} / \mathrm{g}$ for bulk conducting wood and $0.97 \mathrm{~g} / \mathrm{g}$ for pure wood, respectively. It is clearly that the bulk conducting wood has reduced the water adsorption about 33\% than that of the pure wood. This is in good agreement with above-mentioned porosity values showed in Table 1. This is reasonable because the pores of bulk conducting wood should be filled with a lot of PANI molecules.

\section{Conclusions}

In summary, the bulk conducting wood can be fabricated by this work introduced process, e.g., to pre-immerse the wood segment in ANI solution to allow the ANI molecules pre-penetration or diffusion into the wood pore structure, then to start the in situ solution polymerization of PANI in the wood. By this process, the PANI molecules would physically fill into the pore structure of wood and chemically interacted with the $\mathrm{CH}_{2}-\mathrm{OH}$ groups of wood though the glucose units of cellulose. The bulk conducting wood showed expected conductivity and enhanced thermal stability when the temperature over $400^{\circ} \mathrm{C}$.

\section{Compliance with ethical standards}

Conflict of interest The authors declare that they have no conflict of interest.

\section{References}

1. Sjostrom E (1993) Wood chemistry fundamentals and applications. Academic Press, San Diego

2. Song J, Chen C, Zhu S, Zhu M, Dai J, Ray U, Li Y, Kuang Y, Li Y, Quispe N et al (2018) Processing bulk natural wood into a highperformance structural material. Nature 554:224-228

3. Dai S, Wang Y, Zhang J, Zhao Y, Xiao F, Liu D, Wang T, Huang J (2018) Wood-derived nanopaper dielectrics for organic synaptic transistors. ACS Appl Mater Interfaces 10:39983-39991

4. Che W, Xiao Z, Wang Z, Li J, Wang H, Wang Y, Xie Y (2019) Woodbased mesoporous filter decorated with silver nanoparticles for water purification. ACS Sustain Chem Eng 7:5134-5141

5. Guo H, Luković $M$, Mendoza M, Schlepütz CM, Griffa M, Xu B, Gaan S, Herrmann H, Burgert I (2019) Bioinspired struvite mineralization for fire-resistant wood. ACS Appl Mater Interfaces 11:5427-5434

6. Khakalo A, Tanaka A, Korpela A, Hauru LK, Orelma H (2019) Allwood composite material by partial fiber surface dissolution with an ionic liquid. ACS Sustain Chem Eng 7:3195-3202

7. Yang X, Yu YKM, Cho DW, Chen SS, Tsang DCW, Shang J, Yip ACK, Wang L, Ok YS (2019) Tin-functionalized wood biochar as a sustainable solid catalyst for glucose isomerization in biorefinery. ACS Sustain Chem Eng 7:4851-4860

8. Nagasawa C, Kumagai Y (1989) Electromagnetic shielding particle-boards with nickel-plated wood particle. J. Wood Sci 35:1092
9. Nagasawa C, Kumagai Y, Urabe K (1990) Electromagnetic shielding effectiveness particleboard containing nickel-metalized wood particles in the core layer. J. Wood Sci 36:531

10. Nagasawa C, Kumagai Y, Urabe K (1991) Electro-conductivity and electromagnetic shielding effectiveness of nickel-plated veneer. J Wood Sci 37:158

11. Nagasawa C, Umehara H, Koshizaki N (1991) Effects of wood species on electroconductivity and electromagnetic shielding properties of electrolessly plated sliced veneer with nickel. J Wood Sci 40:1092

12. Nagasawa C, Kumagai Y, Koshizaki N, Kanbe JT (1992) Changes in electromagnetic shielding properties of particleboards made of nickel-plated wood particles formed by various pre-treatment processes. J Wood Sci 38:256

13. Nagasawa C, Kumagai Y, Urabe K, Shinagawa SJ (1999) Electromagnetic shielding particle board with nickel-plated wood particles. J Porous Mater 6:247

14. Huang JT, Zhao GJ (2004) Electroless plating of wood. J Beijing For Univ 3:97

15. Wang LJ, Li J, Liu YX (2006) Preparation of electromagnetic shielding wood-metal composite by electroless nickel plating. Mater Sci Technol 6:296

16. Wang LJ, Li J (2007) Ultrasound-assisted electroless plating $\mathrm{Ni}-\mathrm{P}$ alloy on the surface of birch veneer. Sci Silvae Sin 12:311

17. Wang LJ, Li J, Liu YX (2008) Study on preparation of electromagnetic shielding composite by electroless copper plating on Fraxinus mandshurica veneer. J Mater Eng 4:56

18. Kanwal F, Siddiqi SA, Tasleem S, Sakina G, Jamil T (2009) Synthesis and characterization of polyaniline/wood and polyaniline/ carbon composites. J Chem Soc Pak 31:882-887

19. Li J, Wang L, Liu H (2010) A new process for preparing conducting wood veneers by electroless nickel plating. Coat Technol 204:1200-1205

20. Trey S, Jafarzadeh S, Johansson M (2012) In situ polymerization of polyaniline in wood veneers. ACS Appl Mater Interface 4:1760-1769

21. Shen Q, Nylund J, Rosenholm JB (1998) Estimation of the surface energy and acid-base properties of wood by means of wetting method. Holzforschung 52:521-529

22. Shen Q, Mikkola P, Rosenholm JB (1998) Quantitative characterization of the subsurface acid-base properties of wood by using XPS and Fowkes theory. Colloids Surf A 145:235-241

23. Shen Q, Rahiala H, Rosenholm JB (1998) Evaluation of the structure and acid-base properties of bulk wood by FT-Raman spectroscopy. J Coll Interface Sci 206:558-568

24. Dong JQ, Shen Q (2009) Enhancement in solubility and conductivity of polyaniline with lignosulfonate modified carbon nanotube. J Polym Sci B 47:2036-2046

25. Dong JQ, Shen Q (2012) Comparison of the properties of polyaniline doped by lignosulfonates with three different lons. J Appl Polym Sci 126(S1):E10-E16

26. Gu ZJ, Wang JT, Li LL, Chen L, Shen Q (2014) Formation of polyaniline nanotubes with different pore shapes using $\alpha-, \beta$ - and $\gamma$-cyclodextrins as templates. Mater Lett 117:66-68

27. Gu ZJ, Ye JR, Song W, Shen Q (2014) Synthesis of polyaniline nanotubes with controlled rectangular or square pore shape. Mater Lett 121:12-14

28. Shen Q, Zhong L (2007) Lignin-based carbon films and controllable pore size and properties. Mater Sci Eng A 445:731-735

29. Billweyer FW (1994) Textbook of polymer science, 3rd edn. Wiley, New York

30. Gospodinova N, Terlemezyan L (1998) Conducting polymers prepared by oxidative polymerization: polyaniline. Prog Polym Sci 23:1443-1484

31. Hassel BI, Tery S, Leijonmarck S, Johansson M (2014) A study on the morphology, mechanical, and electrical performance 
of polyaniline-modified wood-a semiconducting composite material. BioResources 9:5007-5023

32. Qaiser AA, Hyland MM, Patterson DA (2009) Control of polyaniline deposition on microporous cellulose ester membranes by in situ chemical polymerization. J Phys Chem B 113:14986-14993

33. Renneckar S, Zhou Y (2009) Nanoscale coatings on wood: polyelectrolyte adsorption and layer-by-layer assembled film formation. ACS Appl Mater Interface 1:559-566

34. Shen Q, Zhang T, Zhu MF (2008) A comparison of the surface properties of lignin and sulfonated lignins by FTIR spectroscopy and wicking technique. Colloids Surf A 320:57-60
35. Palaniappan S, John A (2008) Polyaniline materials by emulsion polymerization pathway. Prog Polym Sci 33:732-758

Publisher's Note Springer Nature remains neutral with regard to jurisdictional claims in published maps and institutional affiliations. 